\title{
Apply blockchain technology to electric vehicle battery refueling
}

\author{
Song Hua \\ Fujitsu research \& development \\ center \\ huasong@cn.fujitsu.com \\ Jun Sun \\ Fujitsu research \& development \\ center \\ sunjun@cn.fujitsu.com
}

\author{
Ence Zhou \\ Fujitsu research \& development \\ center \\ zhouence@cn.fujitsu.com \\ Yoshihide Nomura \\ Fujitsu Laboratories \\ y.nomura@jp.fujitsu.com
}

\author{
Bingfeng Pi \\ Fujitsu research \& development \\ center \\ winter.pi@cn.fujitsu.com \\ Hidetoshi Kurihara \\ Fujitsu Laboratories \\ kurihara.hide@jp.fujitsu.com
}

\begin{abstract}
Battery swapping is a solution of electric vehicle (EV) battery refueling. For EV owners, the battery information and transaction's correctness, openness, traceability and immutability is difficult to get guarantee in traditional centralized system. The trust lacking between EV owners and swapping station is caused, and becomes a big challenge to EV's rapid development. An objective mechanism based on decentralized blockchain system is proposed to manage battery swapping and solve the trust lacking issue. With this solution, both battery's life-cycle information and all operations histories are permanently saved in blockchain network. All key logics are driven by smart contracts, the battery price calculation and the digital currency exchange between EV owners and station are realized by smart contracts automatically and accurately. A primary prototype based on Ethereum is analyzed and implemented to illustrate the feasibility of managing battery swapping and refueling based on blockchain system to solve the trust lacking issue.
\end{abstract}

\section{Introduction}

In last decades, with the increasing development of battery technologies and concerns for the environment, electric vehicles (EVs) technologies got rapid development. Large-scale utilization of EV not only makes deep cuts in the release of greenhouse gases, but also reduces the fuel cost for $\mathrm{EV}$ drivers. It enhances power system security and stimulates the development of renewable energy technologies [1], [2].
However, EV drivers have to face the problem of battery refueling on a daily basis. Once the battery runs out, drivers can recharge it in the charging station. And it is also allowed that drivers are able to swap a depleted battery for a fully charged one provided by the charging station and eliminate much of the waiting time. How to make EV drivers fully trust in station grid and how to ensure the fairness and justice about battery swapping procedure are the two important issues. In this paper, we proposed a solution using blockchain, a distributed secure and private ledger of blocks, to address the trust issues for electric vehicle battery swapping application.

For the battery refueling procedure, especially battery swapping between electric vehicle and station grid, there are many factors impacting the fairness of refueling transactions. For example, different battery brands, different charging current, battery wearing state, remaining capacity, etc. In traditional centralized system, all information about battery and transaction were stored unilaterally in central servers. These features make it difficult to ensure the fairness of the transaction, especially for EV owners. As a result, we need an objective mechanism to evaluate battery's quality to guarantee the fairness and justice. Considering the publicity and self-government features of smart contracts, it is the best option.

Blockchain was first introduced in Bitcoin [3], which is a distributed cryptocurrency system allowing users to exchange coins in an anonymous manner. The structure of blockchain is shown in figure 1. Each block contains a number of transactions, and chains together using the previous block's hash value. This kind of structure makes transactions immutable. Blockchain is also a distributed and decentralized system. All data will be duplicated and stored in every 
node. Hence if hackers want to modify one transaction, they must modify all the subsequent blocks with huge majority collusions in Network. [3] All the system participants collaboratively maintain blockchain through verifying and storing new transactions into smart contract will be activated and executed. Selfgovernment and decentralization are the two features of smart contract. Self-government means once smart contract is deployed, it will run automatically. Decentralization means that smart contract is

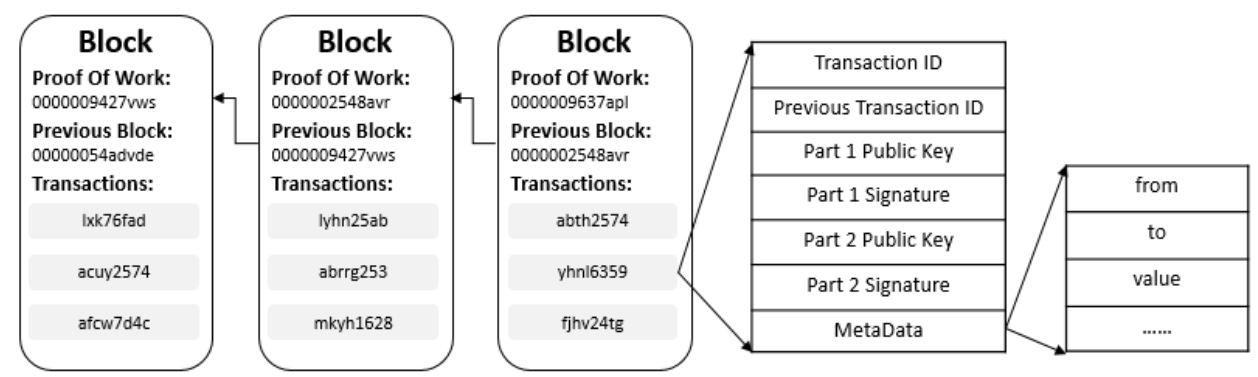

Figure 1. The structure of blockchain

blocks. A transaction is verified by reviewing its signature and checking the previous transaction on the same block. Once verified, the transaction will be added to a block. Once the block size reaches the preset value (block size), the miner of the blockchain network will execute a consensus algorithm to generate a new block. Proof of Work PoW [3] and Proof of Stake PoS [4] are the most popular consensus algorithms. In blockchain, users only show public key to others. So users have anonymous identities. Network participants can use the accordingly public key to verify a transaction and meanwhile protect the privacy. In conclusion, blockchain provides an elegant way to achieve decentralization, makes sure that all transactions are believable and also protects users' privacy.

Smart contracts are executable codes to express logic of transactions in blockchain, such as solidity format in Ethereum [5]. Ethereum blocks thus contain both smart contracts and the final state produced by executing those contracts. The contracts themselves are stored in the blockchain as byte-code instructions for the Ethereum virtual machine (EVM). Several higher-level languages like solidity [6] are available for writing smart contracts. Figure 2 shows the mechanism of the smart contracts. Typically, after all the parties have signed it, smart contract will be attached to the blockchain in the form of program code (such as a record of bitcoin trading data). After P2P network propagation and validated by receiving nodes, smart contract will be deployed into a specific block on the blockchain. Smart contract packages the preset status, transformation rules, execution situation which can be trigged (like arriving specific time or specific event happens), response actions in specific situations and so on. Blockchain can monitor the status of smart contracts in real time. By examining the external data source and make sure specific conditions are triggered, programming code which is stored and verified decentralized. Therefore, smart contract can be used to guarantee the fairness and justice of the transaction.

This paper is organized as follows. Section 2 introduces research progress related to electric vehicle battery refueling. In section 3 , the implementation of applying blockchain for battery refueling is explained in detail. Section 4 discusses the limitation of the proposed solution. Section 5 points out the future work.

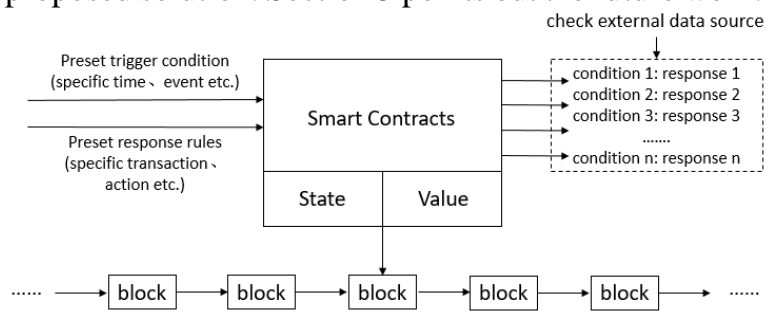

Figure 2. The mechanism of smart contracts

\section{Related Work}

After sweeping over the financial industry, blockchain becomes popular in energy industry [7]. Many blockchain applications have been proposed by energy sectors, such as power purchasing, coal and solar-power trading [7], [8]. These applications provide environmental protection and create new business opportunities.

Due to the limited energy power of EV battery, there is an essential prerequisite to refuel it for long distance driving. Figure 3 shows three methods for EV battery refueling.

1) Alternating Current (AC) charging. Each EV needs a conversion equipment to plug into the $\mathrm{AC}$ outlets. There are various kinds of conversion equipment, which can convert up to $3.3 \mathrm{~kW}, 6.6 \mathrm{~kW}$ or 
$9.6 \mathrm{~kW}$ of power. AC charging is time-consuming, may spend more than 8 hours.

2) Direct Current (DC) charging. DC station grid can get and supply far more power, vary from $25 \mathrm{~kW}$ to $60 \mathrm{~kW}$, or from $90 \mathrm{~kW}$ to $120 \mathrm{~kW}$. DC charging is rapid with about 1 2 hours. However, it
[15], [16]. From these researches we know that current battery swapping process is controlled by the centralized station operators, this mechanism may cause several issues as follows.

1) Short of fair charging evaluation. No matter $\mathrm{AC}$ or DC charging, the charging cost is controlled by

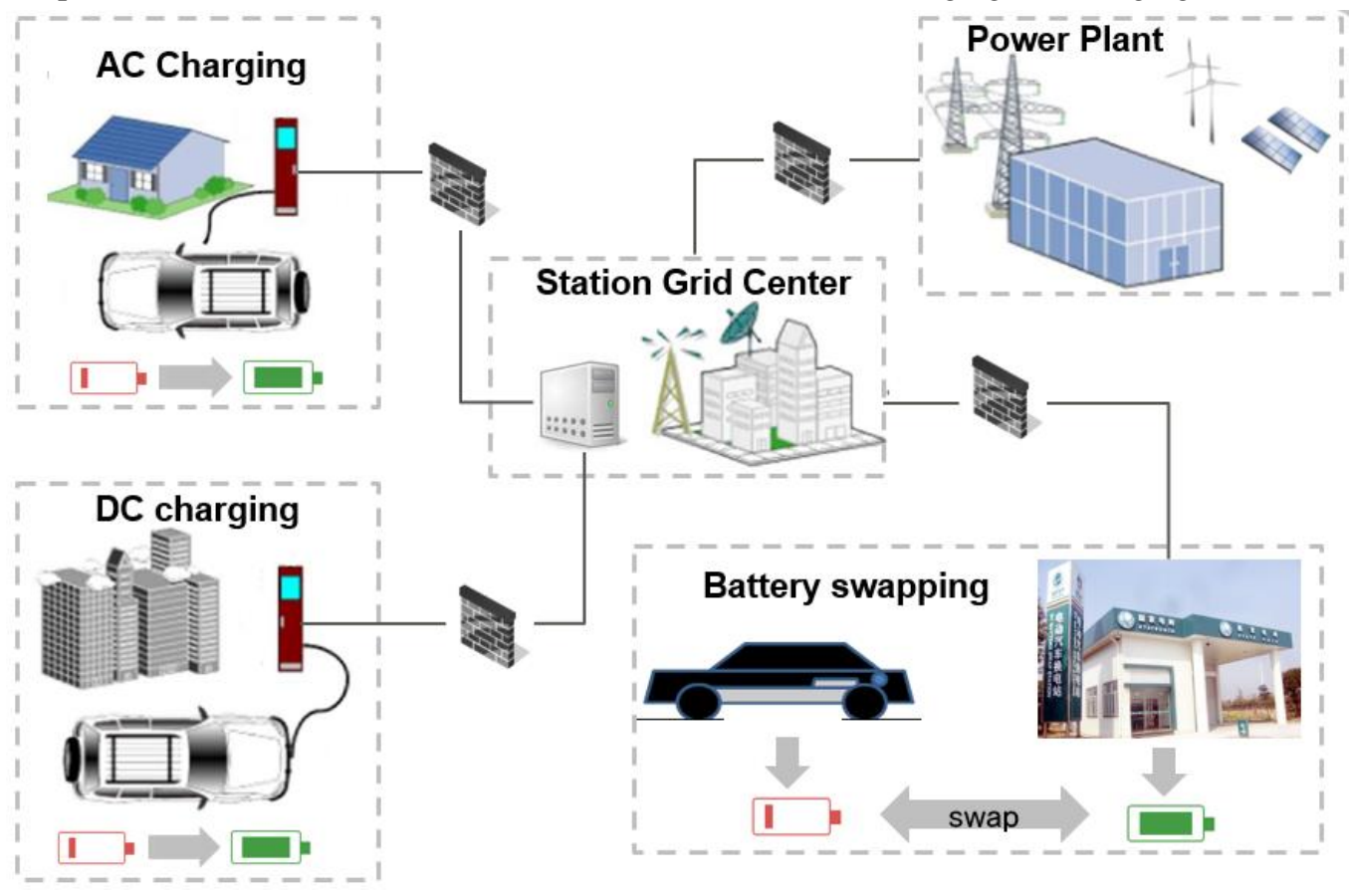

Figure 3. EV battery refueling in a nutshell

may harm EV battery under large power charging.

3) Battery Swapping. Under this mode, the batteries are leased to the EV owners. And EV owners can swap battery with station grid within a few minutes. The swapped empty batteries can be charged during off-peak periods in the station grid.

Around this research area of battery refueling, many research institutions proposed some practical and effective plans. Tesla Motors introduced battery swapping technology for their EVs in 2013 [9]. Some strategies about battery charging have been proposed based on dynamic electricity rates [10]. Some other researchers presented an electric vehicles battery swap stations location routing problem (BSS-EV-LRP), which aims to determine the location strategy of battery swap stations (BSSs) and the routing plan of a fleet of electric vehicles (EVs) simultaneously under battery driving range limitation [11]. Also, several utilities in China installed BSSs for their EV population in 2013 [12], [13]. Yu Zheng et al. proposed a framework for optimal design of battery charging/swapping stations in distribution systems based on life cycle cost (LCC) [14]. Some novel automotive security architectures have been proposed to satisfy the needs of future services of smart vehicles the centralized station grid. EV owner is neither clear about the pricing system nor the battery status after charged.

2) No battery life-cycle management. The initial status of battery before leaving the factory can be viewed as the primary information, such as its manufacturer, construction material, size and power capacity. Besides the primary data, battery's ownership and refueling history are also concerned before swapping. Without these kinds of information, station operators and EV drivers cannot check the health status of battery.

So, in this paper we proposed a solution using blockchain technology to solve the problems about the fairness and justice of trade procedure, as well as the trust issue of battery information.

\section{Implementation of blockchain based battery swapping mode}

This paper implemented a blockchain based demonstration of battery swapping mode (BSM). This application targets for both station operators and EV owners. By using the functions defined in our smart 
contracts, users can manage their own batteries, like carrying out battery swapping. Meanwhile, we have achieved several smart contracts to process battery's information and related operations. Different actions, permissions and frontend UI for EV owners and station operators have been set. In addition, we have also provided a trace module for blockchain account and specific battery.

\subsection{Framework}

Figure 4 shows the construction of BSM. It contains 3 parts: EV part, station part and the Ethereum blockchain side. The EV part and station part depict the actions that can be performed by EV owners and station operators respectively. Both users can buy and register battery. The difference is that station may have more than one battery, while EV driver can have only one. For swapping process, EV owners can submit a swapping request. Once station received this request, the operator can accept or reject it. In the part of Ethereum blockchain, several smart contracts are implemented to manage battery evaluation and swap procedure.

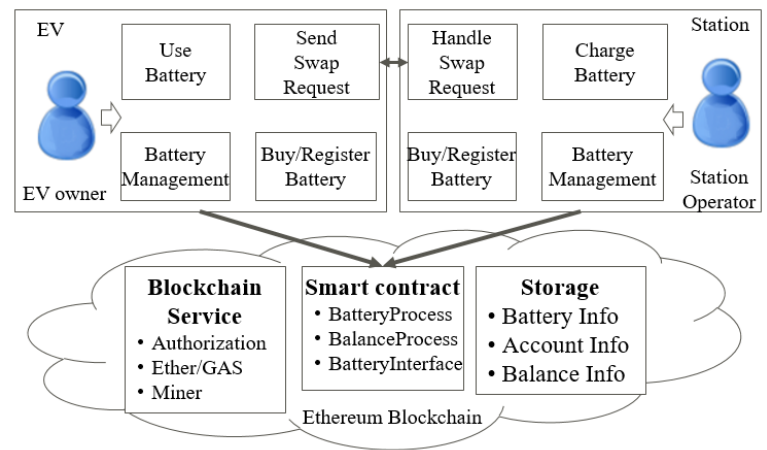

Figure 4. Main construction of BSM

A simple battery swapping scenario is considered as follows.

1) Initially, station operator may buy and register several batteries which belong to this station.

2) If EV owner does not have a battery, he can buy one. Once he already has one battery, he can also register it.

3) EV owner invokes the discharging action to simulate energy consuming, until the battery energy is low.

4) When EV come to a station, EV owner can select a full energy battery from station battery list, and submits a swapping request.

5) Station operator checks swapping request and confirms it.

6) Smart contract calculates the prices of two batteries automatically. If there is a price difference, it will charge coins from one side with lower price battery, and compensate to the other side.

During our implementation, the smart contracts are written in Solidity, and run on Ethereum client.

\subsection{Main processes of BSM}

Currently, we have achieved several main modules of battery swapping mode in our system.

3.2.1. Battery quality evaluation. We think battery's depreciation rate is the most important factor which affects battery's quality. J. Xu [17] have provided a method to evaluate battery's quality based on several depreciation rates. We have made some optimization about these factors as follows:

Time depreciation rate: Each battery has its own service life, even though it has never been used. So time depreciation rate can be seen as a proportion of the time that battery have been used and the time of battery service life. We use formula 1 to calculate time depreciation rate:

$$
\text { Rate }_{\text {Time }}=1-\frac{\text { CurrentMonth-ManufactureMonth }}{\text { MaxMonthLimited }}
$$

Charging \& discharging depreciation rate: Charge and discharge number is a significant factor affecting rechargeable battery. Any kind of rechargeable battery has a specified maximum number of charging and discharging times. Formula 2 and 3 show the method to calculate charging and discharging depreciation rate:

$$
\begin{gathered}
\text { Rate }_{\text {Charging }}=1-\frac{\text { CurrentChargingTimes }}{\text { MaxChargingTimes }} \\
\text { Rate }_{\text {Discharging }}=1-\frac{\text { CurrentDischargingTimes }}{\text { MaxDischargingTimes }}
\end{gathered}
$$

In these two formulas, CurrentChargingTimes and CurrentDischargingTimes mean the already charged and discharged times of battery. Meanwhile MaxChargingTimes and MaxDischargingTimes mean the maximum (rated) number of charging and discharging times.

Load depreciation rate: The load means electric power load of battery. It's related with electric current, voltage and duration of charging / discharging. Load rate can be calculated as a proportion of current load and maximum total load of battery. Formula 4 and 5 is to get the load of charging and discharging:

$$
\text { Load }_{\text {Charging }}=\text { ChargingCurrent } *
$$$$
\text { ChargingVoltage } * \text { ChargingDuration }
$$

$$
\text { Load }_{\text {Discharging }}=\text { DischargingCurrent } *
$$
DischargingVoltage * DischargingDuration (5) 
Then we use formula 6 to calculate the load depreciation rate. In formula 6, MaxLoad Charging and MaxLoad $_{\text {Discharging mean the maximum (rated) total }}$ load of charging and discharging.

$$
\begin{aligned}
& \text { Rate }_{\text {Load }}=1 \text { - }
\end{aligned}
$$

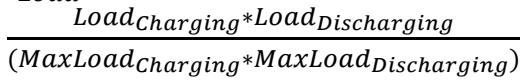

Battery price is calculated based on the evaluation result of battery quality. The multiplication of factory price and battery quality is considered as the main part of battery price. In addition, the remaining energy represented by the state of charge (SoC) is also considered. Formula 7 is used to calculate the battery price:

$$
\begin{aligned}
& \text { Price }=\text { Price }_{\text {Factory }} * \text { Rate }_{\text {Time }} * \text { Rate }_{\text {Charging }} * \\
& \text { Rate }_{\text {Discharging }} * \text { Rate }_{\text {Load }}+\mathrm{SoC} * \text { EnergyPrice }
\end{aligned}
$$

The maximum (rated) service life time, charge \& discharge times and total load of battery may vary with its brand and size. In our BSM system, we allow user to initial these kinds of information when registering new batteries. In real world application, such information must been set by battery manufacturer. It should be kept in a place such as read-only ROM inside of battery.

3.2.2. Charging $\&$ Discharging. The charging action is supported for both station operator and EV owner. Low energy batteries can be charged in station grid during off-peak periods. For EV owner, if time allowed, he can choose a certain station to charge his battery.

EV owner can invoke discharging action to consume electric power of his own battery. However, discharging action is not supported for station operator.

3.2.3. Battery swapping process. Figure 5 shows the main swapping process of BSM.

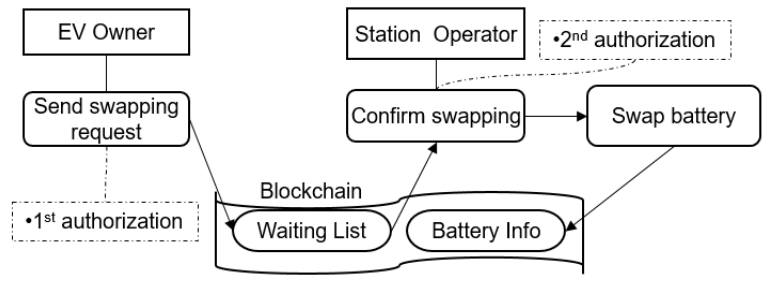

Figure 5. Multiple authorization swapping process

In practical application, the battery swapping process must be authorized by both the owner and the station operator. When EV owner submits a swapping request, it will be sent into a waiting list kept in smart contract. Then station operator can check swapping request from it, and make confirmation.

During these bilateral authorization, both EV owner and station operator should provide the messages which are all signed with their passphrase (or private key) of their accounts.

The multiple authorization swapping process guarantees the consistency of battery ownership.

\subsection{Smart contracts}

For our demonstration, we have implemented 3 smart contracts. Figure 6 shows their relationship.

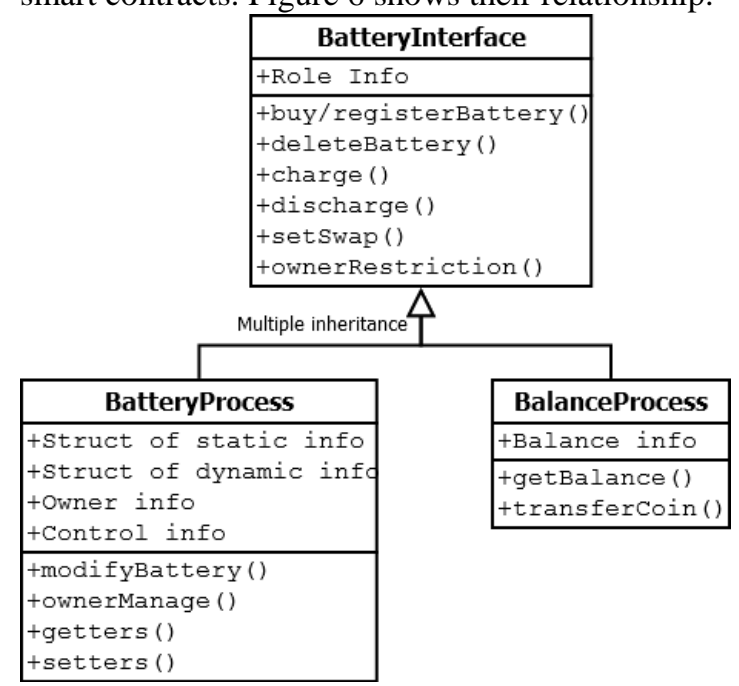

Figure 6. Implementation of smart contracts

BatteryProcess. This contract is used to process battery information. It contains both static and dynamic information of battery. Static information represents battery's properties that can't be altered. For example the manufacture time, brand, factory price. Dynamic information is used to update the battery's status, such as charge times, remained state of charge and so on. Figure 7 shows the detailed properties of static and dynamic information. In addition, the ownership of battery is also concerned. It is used to identify the ownership between battery and account.

This contract also provides a series of relevant operations with battery information, such as information setting and retrieval, and ownership management.

\begin{tabular}{|l|l|}
\hline \multicolumn{1}{|c|}{ Static info } & \multicolumn{1}{|c|}{ Dynamic info } \\
\cline { 2 - 2 } - Battery ID & -Actual charging/discharging times \\
- Brand & -Actual charging/discharging duration \\
- Production time & -Owner account \\
- Max charging/discharging times & -SOC \\
- Manufacture price & - Energy \\
- Max charging/discharging duration & - Price \\
\hline
\end{tabular}

Figure 7. Basic information of battery 
BalanceProcess. It is a contract to manage electric coin of different users. We have defined a token called E-coin as the currency in our system. When user triggers a transaction with currency transferring, such as purchase battery from manufacturer, smart contract will calculate the price. Then it will transfer E-coin margin to the target account automatically.

BatteryInterface. This contract provides API interface for terminal users. Currently there are three different roles of terminal users: station operator, EV owner and super account.

Station operator means the employee of battery station. They can manage batteries which belong to the station. And they must deal with the swapping request which was sent from EV owners.

EV owner can manage his own battery. When he wants to make battery swapping with a station, he can send a request and wait for the confirmation.

Super account is configured to manage the GAS required by each account to invoke smart contract. It is also considered as the battery manufacturer. When station operator and EV owner purchase batteries, they must send certain amount of E-coins to this account.

\subsection{Environment and Experiments}

3.4.1. Deployed Environment. A real and available blockchain environment is generated for our demonstration. Figure 8 is the main deployed environment of our system. We have achieved six Ethereum nodes as the private blockchain network. In these six nodes, there are three miners and three RPC nodes. Mining node mines blocks for the network, meanwhile RPC nodes support RPC service for frontend UI.

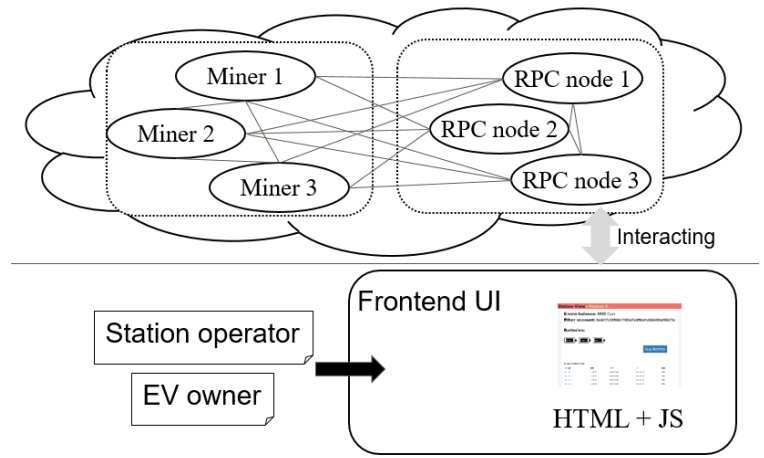

Figure 8. Deployed environment

We use Truffle to develop our system. All frontend JavaScript code is based on Truffle and Ethereum JavaScript API, such as Web3.

In the early stage, we use TestRPC as the test blockchain network, which is very convenience for development and test. For the deploying in real environment, we use Geth as the client of Ethereum to generate all nodes of the network.

3.4.2. Module design. We'll introduce several main modules for station operators and EV owners.

A. For station operators

Index page. This page is the index of station operators. Station operators can check the overview of his own batteries.

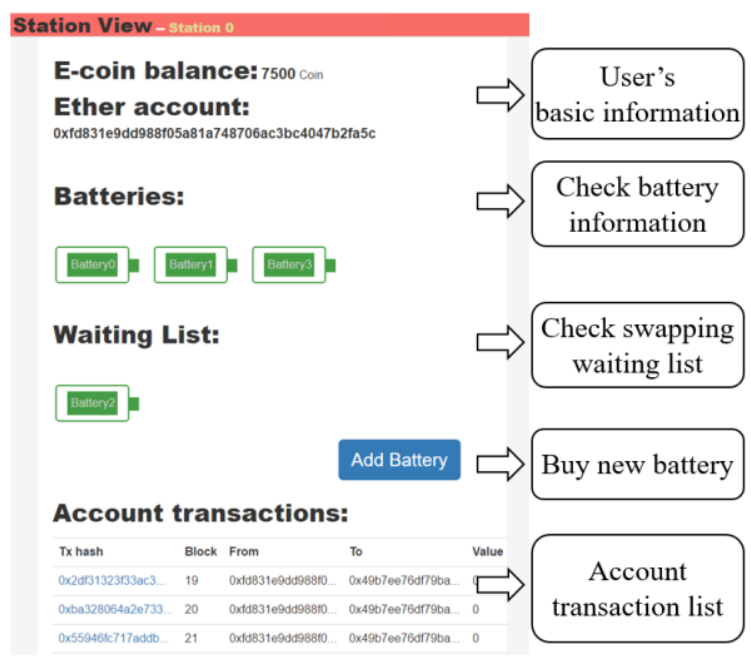

Figure 9. Index page of station operators

Battery detail page. This page is used to check the detailed information about specific battery, including both static and dynamic information, and related transaction list. Station operators can also charge this battery or confirm specific swapping process in this page.

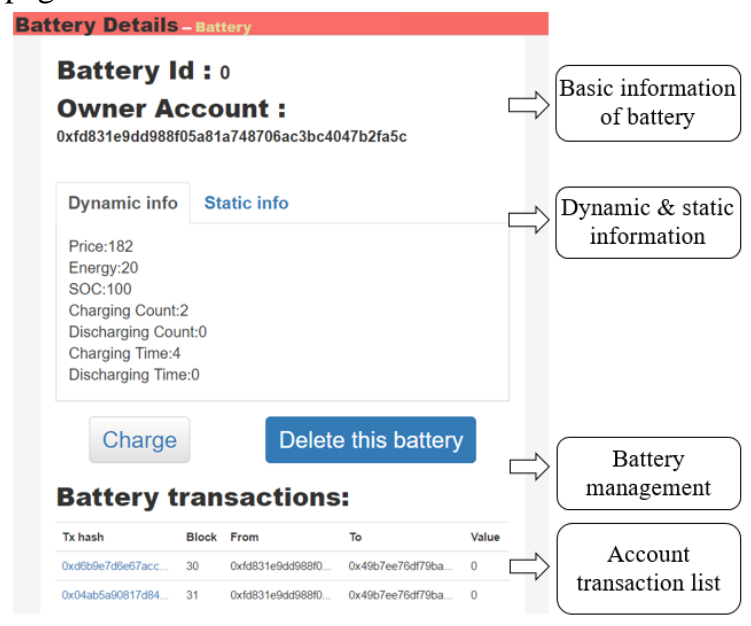

Figure 10. Battery detail page of station operators

B. For EV owners

Index page. The index page of EV owners. Similar to station operators, EV owners can check the 
overview information of himself. Note that EV owners can buy only one battery.

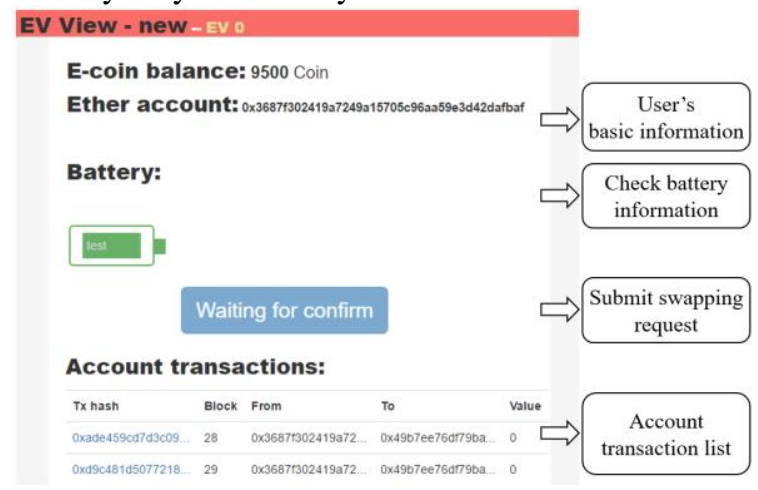

Figure 11. Index page of EV owners

Battery detail page. EV owner also has his own page to check the detailed information of battery. EV owner can use discharging button to simulate driving car and consuming power of battery.

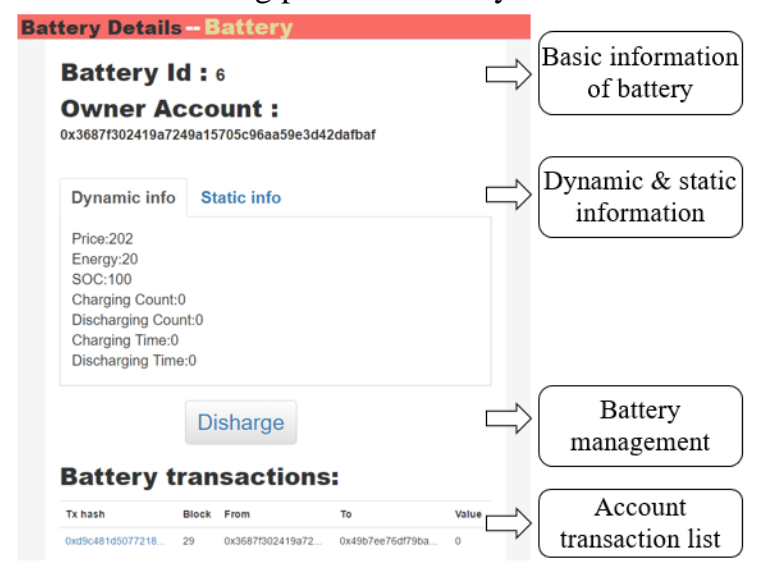

Figure 12. Battery detail page of EV owners

\section{About traceability}

We also present a module to trace a specific battery or a specific account. At the bottom of each page, which is showed in from figure 9 to figure 12 , there is a transaction list component about current account or battery. For index page, it shows all transactions related with the account. And for battery detail page, it shows all transactions related with the battery.

Both station operators and EV owners can check the detailed information of each transaction.

3.4.3 Dynamical simulation. The correctness and feasibility of smart contracts is the most important in battery swapping mode. In order to evaluate the correctness and feasibility of our price and balance module, we have designed a dynamical simulation of iterative operations.
The procedure of simulation follows the steps of battery swapping scenario in section 3.1. To be specific, one iterative circle is:

(1) EV owner invokes the discharging action to simulate energy consuming.

(2) EV owner selects a full energy battery from the battery list of one station, and submits a swapping request. Station and battery are both chosen randomly.

(3) Station operator checks swapping request and confirms it.

(4) Smart contract calculates the prices of two batteries automatically. If there is a price difference, it will charge coins from one side with lower price battery, and compensate to the other side.

Initially, we have set 2 stations and $7 \mathrm{EVs}$, each with 10000 E-coins as their original balances. Each station has four batteries and each EV has only one battery. Based on these simulate steps, we use Python scripts to execute the iterative circle hundreds of times. The program recorded both balance and price information in each circle.

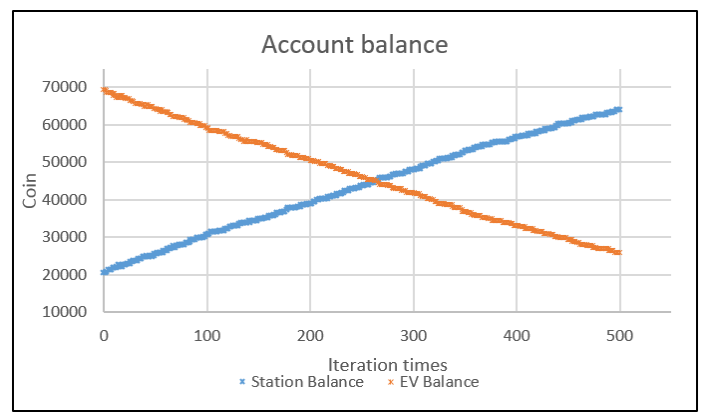

Figure 13. Balance result after 500 times iteration

In figure 13, the blue line is the total balance of 2 stations, meanwhile the orange line is the total balance of 7 EVs. This balance result shows that our balance logical module makes enough sense for users.

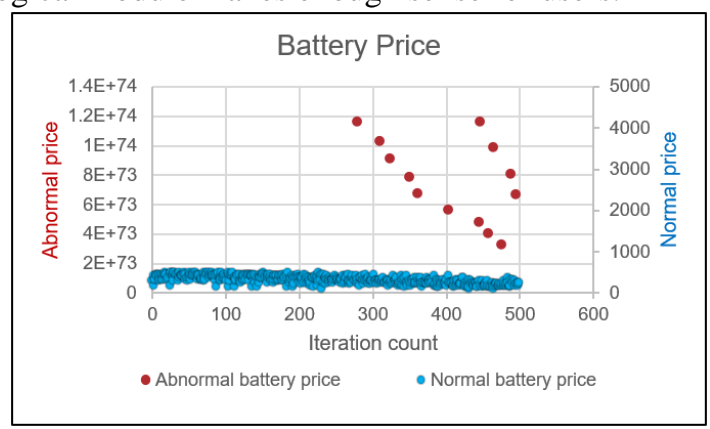

Figure 14. EV received battery price

Figure 14 shows the price curve of EV received battery after swapped with station. The blue points are normal and correct battery prices, while the red points are abnormal prices. The abnormal price value is very high, even more than $1 \mathrm{E}+74$. After checking the source code of smart contracts and debugging, we 
have found that the reason is the overflow of uint when the price is negative.

Because solidity doesn't support float or double types, we have to multiply the numerators by 10000 for retaining the decimal part. We use uint as the type of price and related depreciation rate. For example, the fragment of original source code:

1. uint rateCharging $=10000$ -

currentChargingTimes*10000/maxChargingTimes;

When currentChargingTimes has surpassed maxChargingTimes, the value of rateCharging will be negative, which will cause underflow of uint.

So we have modified the calculation logic code:

1. uint rateCharging $=$

currentChargingTimes*10000/maxChargingTimes;

2. if(rateCharging $>10000)\{$

3. rateCharging $=0$;

4. \}else\{

5. rateCharging $=10000$-rateCharging;

6. \}

The optimized code will check all the depreciation rates to avoid underflow. Furthermore, we will also handle with these batteries which have negative or zero depreciation rate.

Beyond these two simulations about balance and price we carried out, there are other logic modules need to be simulated or tested. We will continue working on this subject in the future.

\section{Summary and discussion}

In 2011, MIT published a paper saying that an EV's battery could cost as much as $25 \%$ to $50 \%$ of the overall cost of a car [18]. As a result, EV owner will be very concerned about the quality of received battery while making swapping with station grid.

Utilizing blockchain technology, we have proposed an objective mechanism to evaluate the quality of battery automatically. Battery related operations are implemented with smart contract. Besides that, the life cycle information of battery is stored in blockchain network, keeping data immutable and traceable. These data are public to both EV owners and stations, to check the healthy status of battery. As a result, it ensures fair transactions between trustless EV owner and station operator.

However, there are some aspects we have not considered very well.

Identification of battery. In blockchain, each battery has a unique virtual identification. All information related with the battery have been mapped to its virtual ID. Ideally, each battery can be attached with an RFID in physic world. However there is no guarantee that battery's RFID information cannot be tampered with. Therefore, we have no idea to ensure the battery identified by RFID is consistent with that of virtual ID.

Maintenance of blockchain. There are 3 kinds of blockchain network in Ethereum: private, consortium and public [19]. For the sake of simplicity, we have deployed and tested on the private blockchain. Both EV drivers and station grids have the rights to be a mining node. However, in practice, EV could not be an Ethereum cluster node due to the limitations of network bandwidth and computing resources. As a result, we should think about alternative authorization method. Actually, consortium blockchain network can also be applicable. IF so, we should consider about how to control the peer nodes. Such as the permission management of peer adding and removing, the mining power maintenance of each peer node.

\section{Conclusion and future work}

Electric vehicle technologies has been developed rapidly in recent years. One of the biggest challenges for large-scale utilizing of $\mathrm{EV}$ is the trust issue between counter parties during battery refueling, especially for battery swapping. Traditional centralized solution is not able to work out this kind of problems.

This paper has proposed a blockchain based decentralized solution for battery refueling. All battery's operations during its life time have been kept in blockchain network. Based on these immutable information of battery, its quality can be evaluated by smart contract automatically. Not only battery's performance degradation over time, but also its depreciation with each recharging cycle have been considered. Therefore, our solution guarantees the fair transaction between trustless of EV driver and station grid.

However, although blockchain and smart contract are fascinating technology, they are still on the proof of concept level. In order to apply our decentralized solution into real word, continuous improvements are required. Such as the monitoring of battery's physical RFID, and the logging and synchronization of various discharging current for different drivers.

Besides that, due to the high cost of distributed network and the immutable of smart contract, it would be best to make a simulation before deploying. Therefore, an important research point is to figure out a solution for simulating real environment.

So far we only considered this battery swapping and refueling solution in terms of real case application. In the next step, we will try to build our theoretical foundation for this topic. Furthermore, we also will develop our own blockchain framework. The 
framework will focuses on 1) Lower cost and higher efficiency network, for example, using new consensus mechanism; 2) Simulating real environment, by collecting more real cases, such as feasible solution of identifying batteries.

\section{References}

[1] A.G. Boulanger, A.C. Chu, S. Maxx, et al., "Vehicle electrification: Status and issues", Journal, Proceedings of the IEEE, 2011, 99(6): 1116-1138.

[2] M. A. Ortega-Vazquez, F. Bouffard, and V. Silva, "Electric vehicle aggregator/system operator coordination for charging scheduling and services procurement", IEEE Transactions on Power Systems, 2013, 28(2): 1806-1815.

[3] S. Nakamoto, "Bitcoin: A peer-to-peer electronic cash system”, white paper, 2008.

[4] G. Wood, "Ethereum: A secure decentralised generalized transaction ledger", Ethereum Project Yellow Paper, 2014.

[5] V. Buterin, "A next-generation smart contract and decentralized application platform", white paper, 2014.

[6] "Solidity documentation", Available: http://solidity.readthedocs.io/-en/latest/index.html.

[7] A. Rutkin, "Blockchain-Based Microgrid Gives Power to Consumers in New York", Available: https://www.newscientist.com/ article/2079334-blockchainbased-microgrid-gives-power-to-consumersin-new-york/, 2016.

[8] E. Munsing, J. Mather, and S. Moura, "Blockchains for Decentralized Optimization of Energy Resources in Microgrid Networks", UC Berkeley: Energy, Controls, and Applications Lab, Available: http://escholarship.org/uc/item/80g5s6df, 2017.

[9] "Tesla Motors: Battery Swap", Available: https://www.tesla.com/videos/battery-swap-event.

[10] O. Worley and D. Klabjan, "Optimization of battery charging and purchasing at electric vehicle battery swap stations", IEEE Vehicle Power and Propulsion Conference, 2011, pp. 1-4.

[11] J. Yang, H. Sun, "Battery swap station location-routing problem with capacitated electric vehicles", Computers \& Operations Research, 2015, 55: 217-232.

[12] "China EV Charging Station and Charging Pile Market Report, 2017-2020”, Research in China, Available: http://www.researchandmarkets.com/reports/4126265/china -ev-charging-station-and-charging-pile\#pos-1, 2017.

[13] "China Electric Vehicle Charging Station and Charging Pile Report 2015-2016”, Research In China, Available: http://www.researchandmarkets.com/reports/3129353/china -electric-vehicle-charging-station-and, 2015.

[14] Y. Zheng, Z. Y. Dong, Y. Xu, K. Meng, J. H. Zhao, and J. Qiu, "Electric vehicle battery charging/swap stations in distribution systems: Comparison study and optimal planning", IEEE Transactions on Power Systems, 2014, 29(1): 221-229.

[15] H. Heinecke, K.P. Schnelle, H. Fennel, et al., "Automotive open system architecture-an industry-wide initiative to manage the complexity of emerging automotive E/E-Architectures", Convergence, 2004, pp.325-332.

[16] M. Steger, C. Boano, M. Karner, et al., "SecUp: Secure and Efficient Wireless Software Updates for Vehicles", IEEE Euromicro Conference on Digital System Design, 2016, pp.628-636.

[17] J. Xu, "Charging exchange method for battery of electric vehicles”, TW201044289A [P]. 2010.12.16

[18] "The Price Of Batteries", Available: https://s3.amazonaws.com/files.technologyreview.com/p/pu b/legacy/jan11_feature_electric_cars_p61.pdf, 2011.

[19] V. Buterin, "Public and private blockchains", Available: https://blog.ethereum.org/2015/08/07/on-public-andprivate-blockchains/, 2015. 